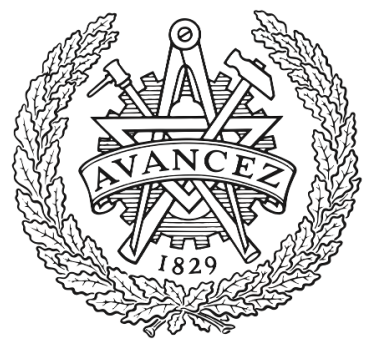

CHALMERS

UNIVERSITY OF TECHNOLOGY

\title{
Longitudinal Force Distribution Using Quadratically Constrained Linear Programming
}

Downloaded from: https://research.chalmers.se, 2023-04-26 09:49 UTC

Citation for the original published paper (version of record):

Klomp, M. (2011). Longitudinal Force Distribution Using Quadratically Constrained Linear

Programming. Vehicle System Dynamics: International Journal of Vehicle Mechanics and Mobility, 49(12): 1823-1836. http://dx.doi.org/10.1080/00423114.2010.545131

N.B. When citing this work, cite the original published paper. 


\title{
Longitudinal Force Distribution Using Quadratically Constrained Linear Programming
}

\author{
M. Klomp* \\ Saab Automobile \\ SE-461 80 Trollhättan
}

\begin{abstract}
In this paper a new method is presented for the optimization of force distribution for combined traction/braking and cornering. In order to provide a general, simple and flexible problem formulation, the optimization is addressed as a quadratically constrained linear programming (QCLP) problem. Apart from fast numerical solutions, different driveline configurations can be included in the QCLP problem in a very straightforward fashion.

The optimization of the distribution of the individual wheel forces using the quasi steady state assumption is known to be useful for the study of the influence of particular driveline configurations on the combined lateral and longitudinal grip envelope of a particular vehicle/driveline configuration. The addition of the QCLP problem formulation, makes another powerful tool available to the vehicle dynamics analyst to perform such studies.
\end{abstract}

\section{Introduction}

Advances in individual distribution of brake and traction forces on all four wheels [6] provide means to enhance the combined longitudinal and lateral grip of the vehicle [12]. This enhancement of the combined grip enables the vehicle to better avoid critical situations, in particular when both longitudinal and lateral forces are simultaneously required. For example when avoiding an obstacle, the traveled distance of the vehicle in a given direction may need

*e-mail: matthijs.klomp@saab.com 
to be minimized or maximized. This is achieved by distributing the forces on each wheel individually such that the global force acting on the center of gravity (vector sum of all tire forces) of the vehicle is maximized in this direction. In optimizing the wheel force distribution also tire and actuator constraints and yaw balance must be considered.

Several studies have been made on how to distribute a predetermined global force and yaw moment to each individual wheel, taking into account actuator and tire constraints. Important contributions in this area are presented in [14], where the force utilization is distributed as equally on all wheels as possible. In [4, 11, 13], additional constraints, due to actuator limitations, are added to such a problem formulation. What is not explicitly considered in [4, 11, 13, however, is that the future tire limits are affected by the instantaneous wheel force distribution because of the lateral and longitudinal load transfer. Therefore, optimality over a longer time horizon is not guaranteed. This issue is addressed using model predictive control (MPC) in for instance [5, 9, 20]. Although all these approaches have their merits for more general problems, they either do not directly consider changes in the tire limits caused by the force distribution, are computationally demanding (non-linear MPC in particular), do not approximate the tire force limits well (when using linearization), or a combination of these.

If, however, the problem being studied represents steady-state conditions, then only the steady-state force distribution needs to be optimized. It is shown in [1 3, 18, that also for smooth changes in the combined longitudinal and lateral acceleration (quasi steady state (QSS) conditions), the lateral and yaw dynamics may be considered to be steady-state. The QSS approximation is used in, for instance, [8] for race car lap simulation and in [16, 17] to optimize the force distribution for cases similar to our present problem.

One particularly relevant reference is [16] where the longitudinal acceleration during traction or braking is maximized for a given cornering radius. This is formulated as a nonlinear optimization problem with the longitudinal force on each wheel as optimization parameters. The lateral forces are subsequently derived from the QSS approximation and the available cornering radius. Nevertheless, in order to more conveniently permit changes in driveline configuration and direction of the optimized force magnitude, the particular problem formulation in [16] needs some adaptation.

The aim of this study is to formulate the force distribution problem in such a way that the direction in which the global force is to be maximized can be easily changed. Another objective is to find a simple and numerically efficient formulation for this problem. Finally, the formulation shall be flexible, such that different driveline configurations and tire constraints can be easily incorporated. 
This paper is organized such that first a simple planar vehicle model is introduced. Following the modeling, the optimization of drive force distribution is formulated as a non-convex quadratically constrained linear programming (QCLP) problem. This approach is applied to four configurations from [17], which are with and without constraints on the left/right longitudinal force distribution. In order to give an example on how the obtained results could be used in a vehicle implementation, a scenario is studied where the global force vector is fixed in the inertial system, demonstrating a wide range of combined braking/traction and cornering. Finally the major findings are summarized and the implications of the results are discussed.

\section{Vehicle Modeling}

The vehicle model used in this study is shown in Figure 1 and is a frontsteered vehicle with one central source of longitudinal force, $F_{X}^{\mathrm{IN}}$. This total longitudinal force is first divided to the front and rear axles and subsequently to each wheel through virtual ideal "differentials" ( $\otimes$ in Figure 1). The difference in drive force between the two outputs is denoted as $\Delta F_{X W 1}, \Delta F_{X W 2}$ and $\Delta F_{X W 3}$ for the front, rear and center differentials, respectively. In the figure, the main part of the nomenclature used for geometry and forces of the vehicle are defined.

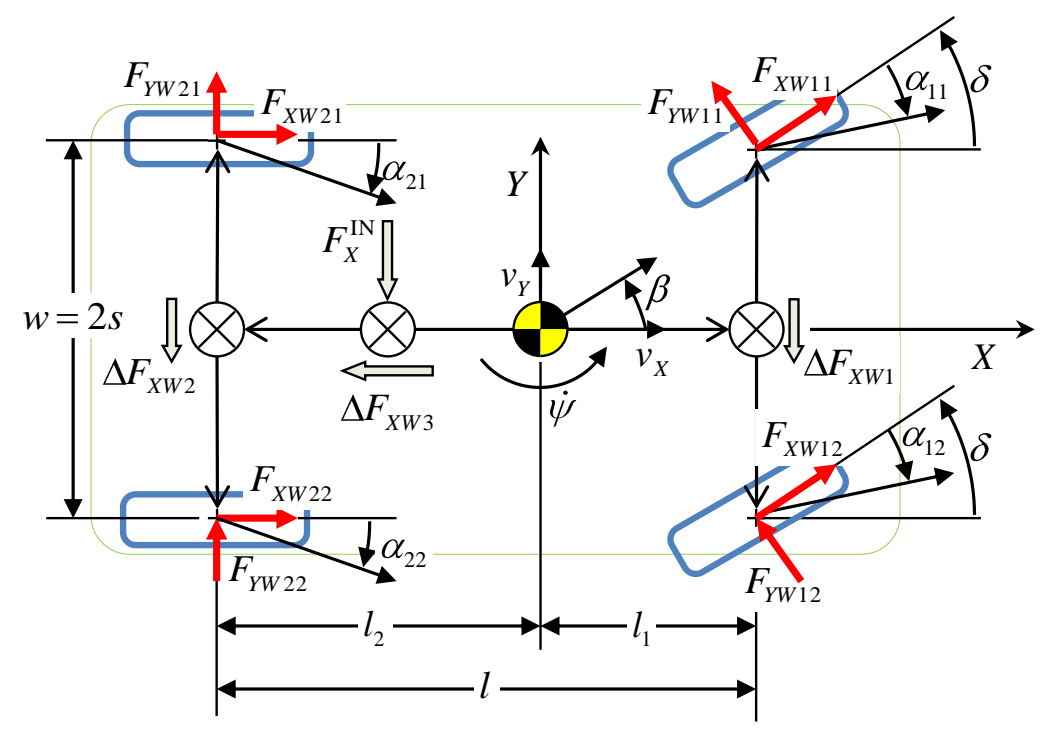

Figure 1: Two-track vehicle model showing the top-view geometry, velocities, slip angles and forces. 


\section{$2.1 \quad$ Two-track Vehicle Model}

In this paper, only the planar motion of the vehicle is considered and the longitudinal, lateral and yaw velocities are chosen as state variables $v_{X}, v_{Y}$ and $\dot{\psi}$, respectively. This means that other dynamics of the vehicle, such as roll, pitch, heave, wheel rotation, steering system and driveline dynamics, are considered rigid. A discussion of some of these assumptions can be found in [19], where the conclusion is that the planar dynamics and tire nonlinearities are the most important factors to consider for most maneuvers. From Newton's second law of motion the following state-space model is derived:

$$
\dot{\boldsymbol{x}}=\mathbf{f}(\boldsymbol{x}, \boldsymbol{u})=\boldsymbol{M}^{-1} \boldsymbol{A} \boldsymbol{z}-\boldsymbol{a}
$$

where the state vector and input vector are

$$
\begin{aligned}
\boldsymbol{x} & =\left[\begin{array}{lllll}
v_{X} & v_{Y} & \dot{\psi}
\end{array}\right]^{\top} \\
\boldsymbol{u} & =\left[\begin{array}{lllll}
F_{X}^{\mathrm{IN}} & \Delta F_{X 1} & \Delta F_{X 2} & \Delta F_{X 3} & \delta
\end{array}\right]^{\top}
\end{aligned}
$$

respectively, and

$$
\begin{aligned}
& \boldsymbol{M}=\operatorname{diag}\left(\begin{array}{lll}
m & m & m k^{2}
\end{array}\right) \\
& \boldsymbol{A}=\left[\begin{array}{lll}
\boldsymbol{p}_{X}^{\top} & \boldsymbol{p}_{Y}^{\top} & \boldsymbol{p}_{\psi}^{\top}
\end{array}\right] \\
& \boldsymbol{p}_{X}=\left[\begin{array}{llllllll}
1 & 0 & 1 & 0 & 1 & 0 & 1 & 0
\end{array}\right]^{\top} \\
& \boldsymbol{p}_{Y}=\left[\begin{array}{llllllll}
0 & 1 & 0 & 1 & 0 & 1 & 0 & 1
\end{array}\right]^{\top} \\
& \boldsymbol{p}_{\psi}=\left[\begin{array}{llllllll}
-s & l_{1} & s & l_{1} & -s & -l_{2} & s & -l_{2}
\end{array}\right]^{\top} \\
& \boldsymbol{z}=\left[\begin{array}{lllll}
F_{X 11} & F_{Y 11} & \cdots & F_{X 22} & F_{Y 22}
\end{array}\right]^{\top} \\
& \boldsymbol{a}=\left[\begin{array}{lll}
-v_{Y} \dot{\psi} & v_{X} \dot{\psi} & 0
\end{array}\right]^{\top}
\end{aligned}
$$

and where $m$ is the vehicle mass, $k$ is the radius of gyration. The lateral forces, expressed in the wheel coordinate systems, $F_{Y W 11} \ldots F_{Y W 22}$, are a function of both the state variables, $\boldsymbol{x}$, and the input vector, $\boldsymbol{u}$. This relationship between the lateral force and the input and state variables is given below. The wheel forces, $\boldsymbol{z}$, are limited according to the friction circle concept [15]. This implies that

$$
F_{X i j}^{2}+F_{Y i j}^{2} \leq \mu_{i}^{2} F_{Z i j}^{2}, \quad \text { for } i=1,2 \text { and } j=1,2
$$

where the indexes $i=1,2$ are for the front and rear axle and $j=1,2$ the left/right wheels, respectively. The vertical forces, $F_{Z i j}$, are given by the 
static load distribution as well as the longitudinal and lateral load transfer [12, 15]:

$$
F_{Z i j}=F_{Z i j}^{0}+(-1)^{i} \zeta_{X} m a_{X}+(-1)^{j} \zeta_{Y i} m a_{X}
$$

where $\zeta_{Y i}$ is the lateral load transfer coefficient of each axle,

$$
\begin{aligned}
F_{Z i j}^{0} & =\frac{\left(l-l_{i}\right) m g}{2 l} \\
\zeta_{X} & =\frac{h}{2 l}
\end{aligned}
$$

and where $h$ is the height of the center of mass above the ground and $g$ is the gravitational acceleration. The longitudinal and lateral accelerations are

$$
\begin{aligned}
& a_{X}=\dot{v}_{X}-v_{Y} \dot{\psi}=\boldsymbol{q}_{X}^{\top} \boldsymbol{z} / m \\
& a_{Y}=\dot{v}_{Y}+v_{X} \dot{\psi}=\boldsymbol{q}_{Y}^{\top} \boldsymbol{z} / m
\end{aligned}
$$

The transformation between the longitudinal and lateral wheel forces expressed in local wheel reference frame and these forces expressed in the vehicle reference frame results in

$$
\boldsymbol{z}=\boldsymbol{T} \boldsymbol{z}_{W}
$$

where

$$
\begin{aligned}
& \boldsymbol{z}_{W}=\left[\begin{array}{lllll}
F_{X W 11} & F_{Y W 11} & \cdots & F_{X W 22} & F_{Y W 22}
\end{array}\right]^{\top} \\
& \boldsymbol{T}=\operatorname{diag}\left(\begin{array}{llll}
\boldsymbol{T}_{1} & \boldsymbol{T}_{1} & \boldsymbol{T}_{2} & \boldsymbol{T}_{2}
\end{array}\right) \\
& \boldsymbol{T}_{1}=\left[\begin{array}{cc}
\cos \delta & -\sin \delta \\
\sin \delta & \cos \delta
\end{array}\right] \\
& \boldsymbol{T}_{2}=\left[\begin{array}{ll}
1 & 0 \\
0 & 1
\end{array}\right]
\end{aligned}
$$

In order to more easily relate the presented results to an actual driveline, the total force and force differences are presented as functions of the longitudinal acceleration. The total longitudinal force and the longitudinal force differences relate to the wheel forces as

$$
\tilde{\boldsymbol{u}}=\boldsymbol{R} \boldsymbol{z}_{W}
$$

where

$$
\begin{aligned}
\tilde{\boldsymbol{u}} & =\left[\begin{array}{cccccccc}
F_{X}^{\mathrm{IN}} & \Delta F_{X 1} & \Delta F_{X 2} & \Delta F_{X 3}
\end{array}\right]^{\top} \\
\boldsymbol{R} & =\left[\begin{array}{cccccccc}
1 & 0 & 1 & 0 & 1 & 0 & 1 & 0 \\
-1 & 0 & 1 & 0 & 0 & 0 & 0 & 0 \\
0 & 0 & 0 & 0 & -1 & 0 & 1 & 0 \\
-1 & 0 & -1 & 0 & 1 & 0 & 1 & 0
\end{array}\right]
\end{aligned}
$$


In summary, the model (1) is a dynamic model on state-space form of a three-degree of freedom all-wheel drive vehicle with three state variables and five input variables.

\subsection{Tire Model}

A simple version of the well-known Magic Formula tire model [15] is used to compute the lateral forces. In this model the longitudinal force, $F_{X W i j}$, and the slip angle, $\alpha_{i j}$, on each wheel chosen as independent variables. The slip angles, $\alpha_{i j}$, are the angles of the velocity vector at each individual wheel. These are obtained from a linear transformation from the longitudinal, lateral and yaw velocity at the center of gravity to the local velocities at the wheels, such that

$$
\alpha_{i j}=\delta_{i}-\arctan \frac{v_{Y}-(-1)^{i} l_{i} \dot{\psi}}{\left|v_{X}+(-1)^{j} s \dot{\psi}\right|}
$$

The lateral forces are given by the Magic Formula:

$$
F_{Y W i j}=D_{i j} \sin \left(\left(C \arctan \left(B \alpha_{i j}\right)\right)\right.
$$

with coefficients

$$
D_{i j}=\sqrt{\left(\mu_{i} F_{Z i j}\right)^{2}-F_{X W i j}^{2}}, \quad F_{X W i j} \leq \mu_{i} F_{Z i j} \cos \alpha_{i j}
$$

and where the friction coefficients, $B$ and $C$ are given in Appendix A. It follows from Equation (13) that the maximum lateral force is achieved for the slip angle:

$$
\alpha_{i j}^{*}=\frac{1}{B} \tan \frac{\pi}{2 C}
$$

\section{Optimizing the Wheel Force Distribution for Maximum Combined Grip}

In this section, the distribution of the individual wheel forces is optimized such that the the magnitude of the global force with a predetermined angle, $\phi$ (relative to the longitudinal direction of the vehicle), is maximized. This problem is formulated as a quadratically constrained linear programming (QCLP) problem which is a special case of quadratically constrained quadratic programming (QCQP) problems [7]. In this section, solutions to the QCLP problem are shown for four different driveline configurations. In the case the power source is, for instance, an electric motor, both traction and braking is possible. For this reason, also braking is considered for the driveline configurations which are studied. 


\subsection{Quadratically Constrained Linear Programming}

The objective to maximize the global force vector in a particular direction $\phi$, subject to tire constraints (4), the yaw balance (1), as well as additional (optional) constraints on the longitudinal force distribution result in the following QCLP problem:

$$
\begin{array}{ll}
\text { minimize } & \boldsymbol{p}_{00}^{\top} \boldsymbol{z} \\
\text { subject to } & \boldsymbol{z}^{\top} \boldsymbol{Q}_{i j} \boldsymbol{z}+\boldsymbol{p}_{i j}^{\top} \boldsymbol{z}+r_{i j} \leq 0 \\
& \boldsymbol{p}_{\psi}^{\top} \boldsymbol{z}=0 \\
& \boldsymbol{E R} \boldsymbol{z}=0
\end{array}
$$

where

$$
\boldsymbol{p}_{00}=-\left(\boldsymbol{p}_{X} \cos \phi+\boldsymbol{p}_{Y} \sin \phi\right)
$$

The objective function maximizes the sum of the wheel forces in the predetermined direction and the (quadratic) inequality constraints are that the tire forces must lie within the friction circle. The first equality constraint is the yaw moment balance that must be maintained. Constraints which are imposed by different driveline configurations are included by changing the matrix $\boldsymbol{E}$.

The inequality constraints for each tire, expressed with $\boldsymbol{z}$, are obtained by combining the Equations (4), (5) and (7):

$$
\boldsymbol{z}^{\top} \boldsymbol{P}_{i j} \boldsymbol{z} \leq \mu_{i}^{2}\left(F_{Z i j}^{0}+(-1)^{i} \zeta_{X} \boldsymbol{p}_{X}^{\top} \boldsymbol{z}+(-1)^{j} \zeta_{Y i} \boldsymbol{p}_{Y}^{\top} \boldsymbol{z}\right)^{2}
$$

where

$$
\begin{aligned}
& \boldsymbol{P}_{11}=\operatorname{diag}\left(\begin{array}{llllllll}
1 & 1 & 0 & 0 & 0 & 0 & 0 & 0
\end{array}\right) \\
& \boldsymbol{P}_{12}=\operatorname{diag}\left(\begin{array}{llllllll}
0 & 0 & 1 & 1 & 0 & 0 & 0 & 0
\end{array}\right) \\
& \boldsymbol{P}_{21}=\operatorname{diag}\left(\begin{array}{llllllll}
0 & 0 & 0 & 0 & 1 & 1 & 0 & 0
\end{array}\right) \\
& \boldsymbol{P}_{22}=\operatorname{diag}\left(\begin{array}{llllllll}
0 & 0 & 0 & 0 & 0 & 0 & 1 & 1
\end{array}\right)
\end{aligned}
$$

By expanding Equation (18) we identify

$$
\begin{aligned}
\boldsymbol{Q}_{i j} & =\boldsymbol{P}_{i j}-\left[\begin{array}{ccc}
\boldsymbol{O}_{i j} & \cdots & \boldsymbol{O}_{i j} \\
\vdots & \ddots & \vdots \\
\boldsymbol{O}_{i j} & \cdots & \boldsymbol{O}_{i j}
\end{array}\right] \\
\boldsymbol{p}_{i j} & =-2 \mu_{i}^{2} F_{Z i}^{0}\left((-1)^{i} \zeta_{X} \boldsymbol{p}_{X}+(-1)^{j} \zeta_{Y i} \boldsymbol{p}_{Y}\right) \\
r_{i j} & =-\left(\mu_{i} F_{Z i}^{0}\right)^{2}
\end{aligned}
$$

where

$$
\boldsymbol{O}_{i j}=\mu_{i}^{2}\left[\begin{array}{cc}
\zeta_{X}^{2} & (-1)^{i+j} \zeta_{X} \zeta_{Y i} \\
(-1)^{i+j} \zeta_{X} \zeta_{Y i} & \zeta_{Y i}^{2}
\end{array}\right]
$$


It may be noted that, since $Q_{i j}$ is neither positive nor negative (semi)definite, the problem is non-convex [7].

The gradients of the inequality and equality constraints are easily identified from the QCLP formulation in Equation (29). These gradients can be supplied to the numerical solver, eliminating the need for these to be estimated by the solver, for faster computations. The gradient of the linear parts of Equation (29) are simply the vectors (or matrix) with which $\boldsymbol{z}$ is multiplied, and for the quadratic inequality constraints

$$
\nabla\left(\boldsymbol{z}^{\top} \boldsymbol{Q}_{i j} \boldsymbol{z}\right)=\boldsymbol{z}^{\top}\left(\boldsymbol{Q}_{i j}+\boldsymbol{Q}_{i j}^{\top}\right)
$$

Summarizing, a QCLP problem (29) is formulated with the objective to maximize the combined grip subject to tire, yaw balance and driveline configuration constraints. This problem is solved with a standard non-linear programming solver (fmincon from the MathWorks ${ }^{\mathrm{TM}}$ ). In order to quantify the benefits of the present formulation with regard to computational efficiency, one example problem when $\boldsymbol{E}=0$ is studied for three different problem formulations, using the same numerical solver. These problem formulations are a standard non-linear programming (NLP) formulation using the constraints in Equation (18) and the QCLP formulation (29) with and without supplying the gradients to the solver. Relative to the NLP formulation, the QCLP problem was nearly three times faster and five times faster if additionally the gradients of the objective function and the constraints were supplied to the solver. It is likely that additional computational benefits are achieved if a specialized solver for QCLP problems is used.

\subsection{Driveline Configurations Using Linear Constraints}

The driveline configurations which are studied are taken from [17] and represent different combinations of constraints on the left/right longitudinal force distributions. Either the left/right longitudinal force distribution is fixed and equal (open) or is free within the tire constraints. These two possibilities per axle are denoted as "o" for open or "a" for active in the index of the $E$-matrix. These configurations impose the following additional equality constraints on the optimization problem such that the $\boldsymbol{E}$-matrix for each configuration becomes

$$
\begin{aligned}
\boldsymbol{E}_{a a} & =0 \\
\boldsymbol{E}_{a o} & =\left[\begin{array}{llll}
0 & 1 & 0 & 0
\end{array}\right] \\
\boldsymbol{E}_{o a} & =\left[\begin{array}{llll}
0 & 0 & 1 & 0
\end{array}\right] \\
\boldsymbol{E}_{o o} & =\left[\begin{array}{llll}
0 & 1 & 0 & 0 \\
0 & 0 & 1 & 0
\end{array}\right]
\end{aligned}
$$


It may be noted that the front/rear drive force distribution is optimized for all four configurations. Although not pursued in this study, it is straightforward to use the $\boldsymbol{E}$-matrix to add a (linear) constraints on the front/rear drive force distribution as well. In this way, front-wheel, rear-wheel or fixed torque-split all-wheel drive configurations can be studied, as in [17]. This is done by adding the row

$$
\boldsymbol{E}_{\mathrm{F} / \mathrm{R}}=\left[\begin{array}{llll}
\xi & 0 & 0 & 1
\end{array}\right]
$$

where

$$
\xi=-\frac{\Delta F_{X W 3}}{F_{X}^{\mathrm{IN}}}
$$

For example, for front-wheel-drive (FWD) $\xi=1$ and for rear-wheel-drive (RWD) $\xi=-1$. For an all-wheel-drive (AWD) configuration with a 50/50 front/rear torque split, $\xi=0$. These additional examples are mentioned to demonstrate the flexibility of the employed formulation to incorporate a wide variety of driveline configurations, beyond the four configurations studied in this section.

\subsection{Solutions for the Different Driveline Configura- tions}

The results from the four different driveline configurations above are shown in Figures 2 and 3 . The vehicle data used for these simulations is given in Appendix A.

In Figure 2 the optimal left/right and front/rear drive force distribution is shown. In this figure, the sub-figures 2. a and 2.b show the optimal left/right longitudinal force differences for front and rear axle, respectively.

Figure 2.c shows the optimal front/rear distribution. The lines along which all the longitudinal force is applied on the front or rear axle are indicated in this sub-figure with diagonal dashed lines indicated with FWD and RWD, respectively.

The maximum combined lateral and longitudinal acceleration is shown in Figure 3. The dashed boundaries in Figure 2.c indicate the traction or braking limit of the front and rear axles.

The results shown in these figures are identical to those presented in [17, where an excellent discussion on the implications of this drive force distribution can be found. 
(a)

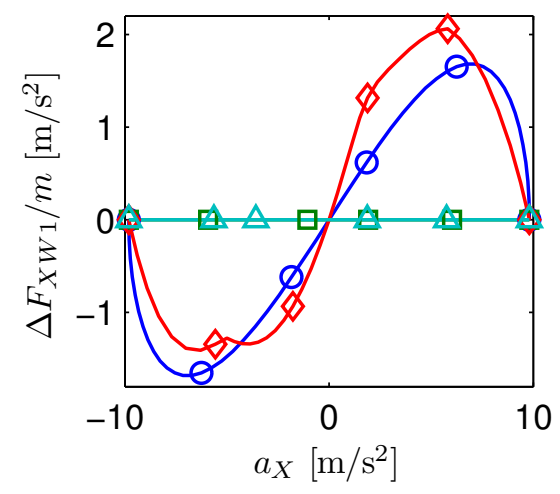

(b)

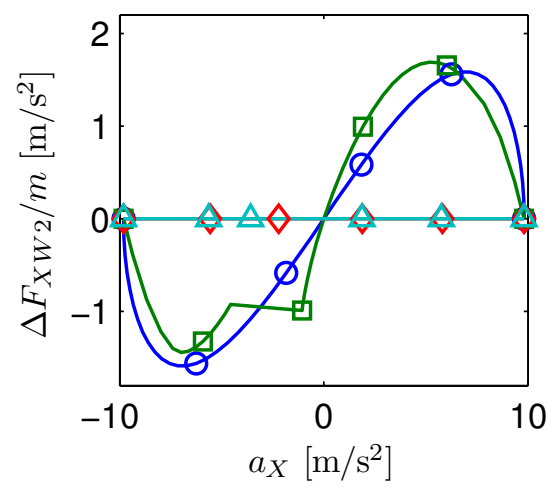

c)

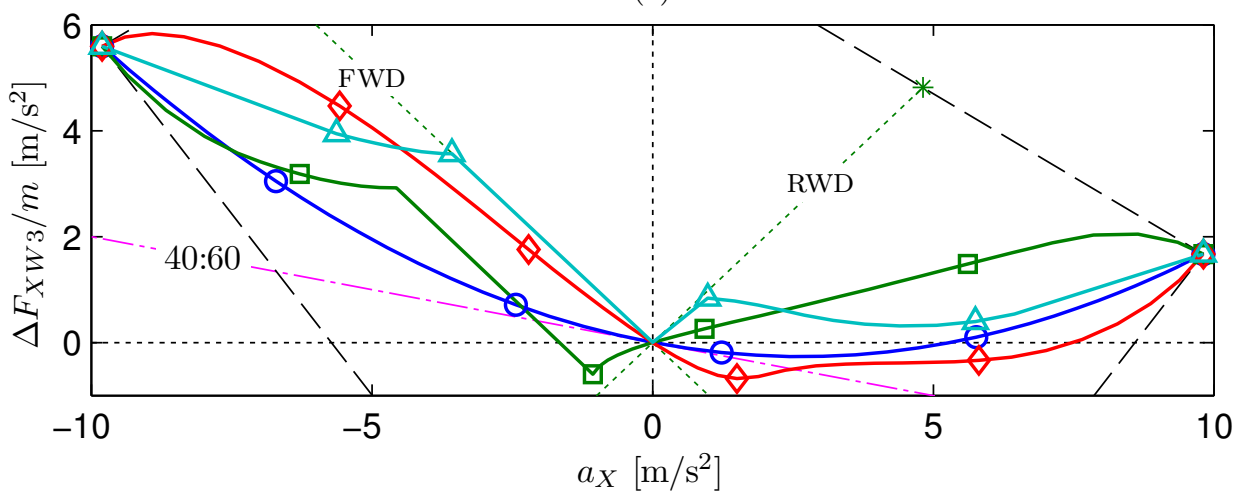

Figure 2: Optimal drive force distribution for four different configurations: active/active (circle), active/open (square), open/active (diamond), open/open (triangle). Results are for $\delta=0$.

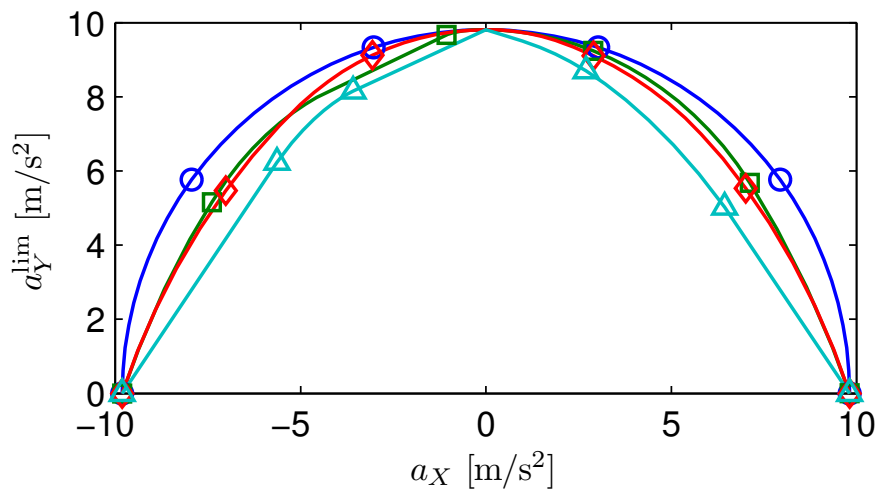

Figure 3: g-g Diagrams for four different configurations: active/active (circle), active/open (square), open/active (diamond), open/open (triangle). 


\subsection{Analytical Solution for Free Longitudinal Force Distribution (active/active)}

Next, closed-form expressions are presented for the case where the longitudinal force distribution can be freely distributed to all four wheels and the friction coefficient, $\mu$, is equal on all four wheels. The proposition is that the friction force capability on each tire should be fully utilized and applied in the same direction as the desired global force direction. It is further suggested that the total friction capability can also be modeled as a circle. This proposition implies that

$$
\left[\begin{array}{c}
F_{X i j}^{*} \\
F_{Y i j}^{*}
\end{array}\right]=\mu F_{Z i j}\left[\begin{array}{c}
\cos \phi \\
\sin \phi
\end{array}\right]
$$

and

$$
\left[\begin{array}{l}
a_{X} \\
a_{Y}
\end{array}\right]=\mu g\left[\begin{array}{l}
\cos \phi \\
\sin \phi
\end{array}\right]
$$

which, when inserted into Equation (5), gives that

$$
\left[\begin{array}{l}
F_{X i j}^{*} \\
F_{Y i j}^{*}
\end{array}\right]=\mu\left(F_{Z i j}^{0}+\mu m g\left((-1)^{i} \zeta_{X} \cos \phi+(-1)^{j} \zeta_{Y i} \sin \phi\right)\left[\begin{array}{c}
\cos \phi \\
\sin \phi
\end{array}\right]\right.
$$

where the asterisk indicates that this is the optimal force distribution for this particular configuration.

In order to verify the proposition, the drive force distribution given by Equation (28) is compared to numerically computed solutions to Equation (29) for this configuration.

In Figure 4 the numerical solution is shown with a solid line and the closed-form solution with circles. From the figure it can be seen that the proposed closed-form solution yields the same results as the numerical solution.

\subsection{Linear Approximation of the Quadratic Constraints}

Although the QCLP formulation has a linear cost function, the QCLP cannot be solved with a standard linear programming solver. In [10], approximations of the quadratic constraints to a polygon are suggested. Using this approximation, the problem is a proper linear programming problem with linear constraints (here denoted LCLP). As mentioned in the introduction, however, the approximation proposed in [10] does not explicitly consider changes in the friction circles as function of the wheel force allocation. In order to address this issue, the same normal force distribution model (5) will be used 
(a)

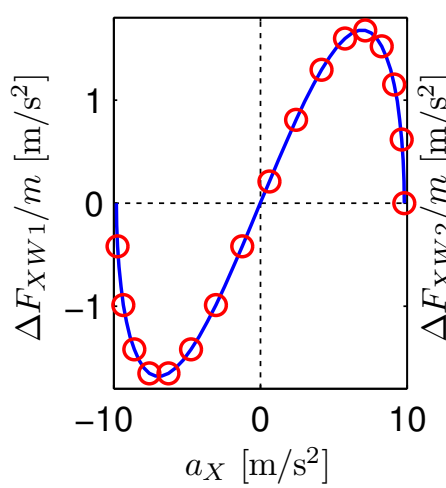

(b)

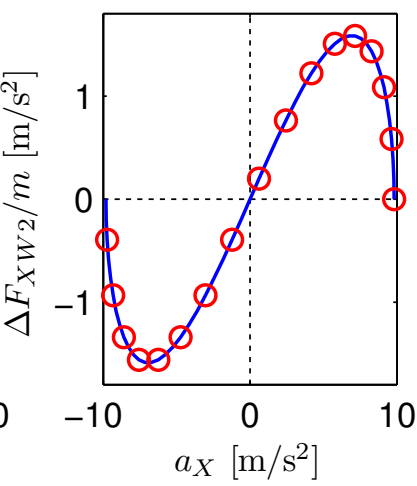

(c)

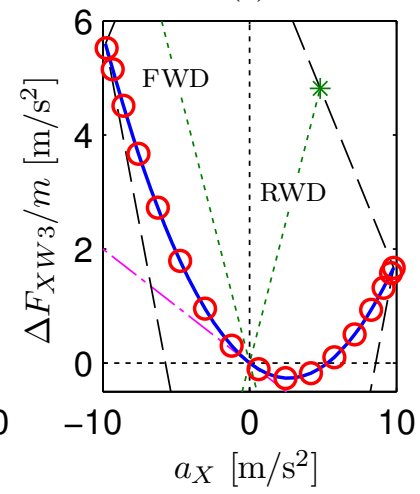

Figure 4: Comparison between numerical (solid line) solution to the QCLP problem 29) and closed-form expressions (circles) in Equation (28).

for the LCLP formulation in the same way as it was done for the QCLP formulation.

With the quadratic constraints being approximated with an octagon, the problem is reformulated to the following LCLP problem:

$$
\begin{array}{ll}
\text { minimize } & \boldsymbol{p}_{00}^{\top} \boldsymbol{z} \\
\text { subject to } & \boldsymbol{D} \boldsymbol{z} \leq \boldsymbol{b} \\
& \boldsymbol{D}_{\mathrm{eq}} \boldsymbol{z}=0
\end{array}
$$

where

$$
\begin{aligned}
& \boldsymbol{D}=\operatorname{diag}\left(\boldsymbol{D}_{0}, \boldsymbol{D}_{0}, \boldsymbol{D}_{0}, \boldsymbol{D}_{0}\right)-\left[\begin{array}{lllllll}
\boldsymbol{d}_{11} & \cdots & \boldsymbol{d}_{11} & \cdots & \boldsymbol{d}_{22} & \cdots & \boldsymbol{d}_{22}
\end{array}\right]^{\top} \\
& \boldsymbol{D}_{0}=\left[\begin{array}{cccc}
1 & \sqrt{2}-1 & 1-\sqrt{2} & -1 \\
\sqrt{2}-1 & 1 & 1 & \sqrt{2}-1
\end{array}\right]^{\top} \\
& \boldsymbol{d}_{i j}=\mu_{i}\left((-1)^{i} \boldsymbol{\zeta}_{X} \boldsymbol{p}_{X}+(-1)^{j} \boldsymbol{\zeta}_{Y i} \boldsymbol{p}_{Y}\right) \\
& \boldsymbol{b}=\left[\begin{array}{lllllll}
\mu_{1} F_{Z 11}^{0} & \cdots & \mu_{1} F_{Z 11}^{0} & \cdots & \mu_{2} F_{Z 22}^{0} & \cdots & \mu_{2} F_{Z 22}^{0}
\end{array}\right]^{\top} \\
& \boldsymbol{D}_{\mathrm{eq}}=\left[\begin{array}{lll}
\boldsymbol{p}_{\psi} & \boldsymbol{p}_{0} & (\boldsymbol{E R})^{\top}
\end{array}\right]^{\top} \\
& \boldsymbol{p}_{0}=\boldsymbol{p}_{X} \sin \phi-\boldsymbol{p}_{Y} \cos \phi
\end{aligned}
$$

This particular problem formulation is only valid for positive lateral forces, since only the left sides of the polygons are given in $\boldsymbol{D}_{0}$. Since left and right cornering are symmetric, extension to right cornering is straightforward but left out for sake of brevity. It is of course also straightforward to extend the above to polygons with more sides than eight.

The additional constraint $\boldsymbol{p}_{0}=0$ is necessary to force the force vector to be in the intended direction. Without this constraint, the solution to the 


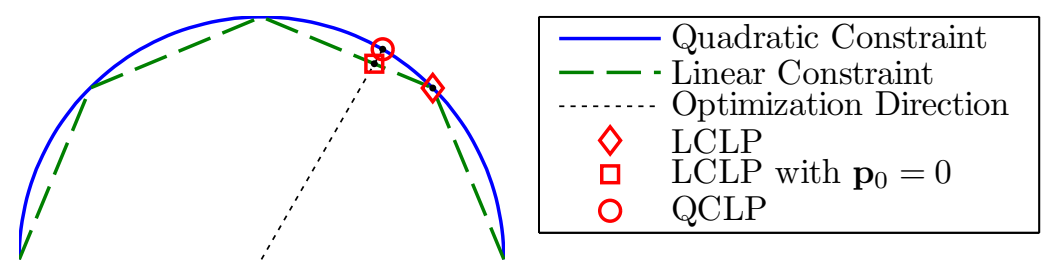

Figure 5: Comparison between QCLP (circles) and LCLP (squares).

(a)

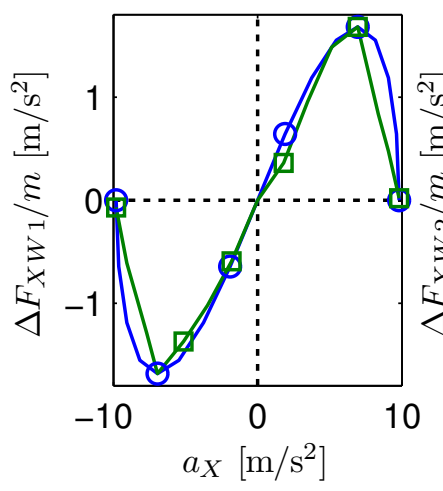

(b)

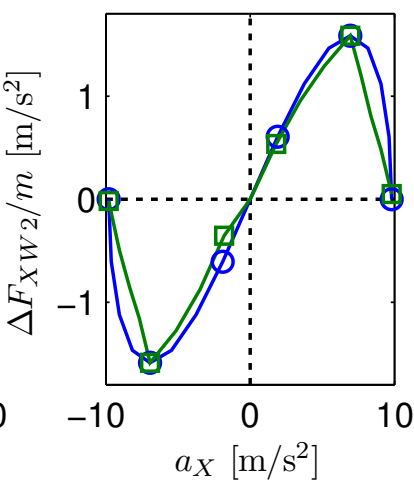

(c)

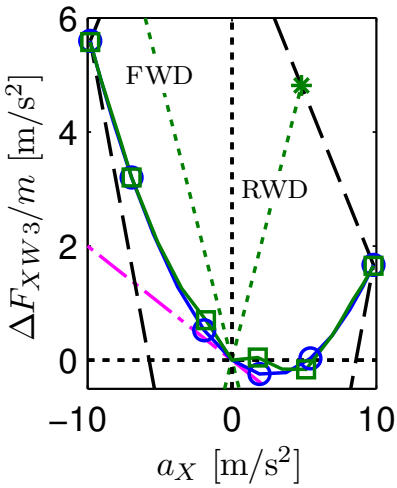

Figure 6: Comparison between QCLP (circles) and LCLP (squares).

LCLP problem will only yield results in the vertexes of the polygons. This is illustrated in Figure 5 where it can be seen that it is always a vertex that has the largest distance in the optimization direction. The only exception when optimizing perpendicular to a side of the polygon; in this case any point along the polygon yields the same result. The additional constraint solves both of these issues.

A comparison for the active/active configuration $(\boldsymbol{E}=0)$ is made between the QCLP formulation and the LCLP formulation for $0 \leq \phi \leq \pi$. The results are shown in Figure 6 and show a reasonable agreement between the two solutions. The main benefit is that solving the LCLP problem with a dedicated LP solver (in this case, linprog from the MathWorks ${ }^{\mathrm{TM}}$ ) was five times faster than the solving the QCLP problem. 


\section{Vehicle Implementation of the QCLP Optimization Results}

The purpose of this section is to given an example of a vehicle implementation of the optimal drive force distribution obtained above. Since we are interested in situations that involve smooth changes in combined longitudinal and lateral acceleration, consider an example where the global force has a fixed direction in the inertial reference frame. With initial conditions

$$
\boldsymbol{x}_{0}=\left[\begin{array}{lll}
15 & 0 & -\pi / 4
\end{array}\right]^{\top}
$$

it can be seen that the desired direction of the global force is $135^{\circ}$ relative to the initial heading of the vehicle. The task is to maximize this global force, resulting in both longitudinal and lateral forces, with the latter turning the vehicle. As the vehicle turns, so does the direction of the global force in the vehicle coordinate system.

The implementation was made by determining the total and longitudinal force difference in the input vector $\tilde{\boldsymbol{u}}$ by linear interpolation of the off-line computed optimal longitudinal force distribution. Since the lateral forces cannot be directly controlled through the input vector, $\boldsymbol{u}$, but only via the front steering angle $\delta$, the inverse of the employed tire model is used to compute the steering input that maximizes the lateral force. The steering angle is computed by combining the average slip angle on the front axle 112 with the slip angle at which the maximum lateral force is achieved (15) from which we obtain

$$
\delta=\alpha_{1}^{*}+\beta_{1}
$$

where

$$
\beta_{1}=\arctan \frac{v_{Y}+l_{1} \dot{\psi}}{\left|v_{X}\right|}
$$

which is the average slip angle on the front axle if $\delta=0$.

Results are shown in Figure 7. In Figure 7, a, the speed history $(v=$ $\left.\sqrt{v_{X}^{2}+v_{Y}^{2}}\right)$ is shown and in 7 . $\mathrm{b}$, the lateral versus the longitudinal acceleration is shown, which can be compared to the g-g diagram in Figure 3. In Figure 7.c, the trajectory in the inertial reference frame is shown. The circles at the location at each wheel indicates the relative size of the friction circle and the bold line within each circle indicates the force vector of that wheel.

From Figure 7.c it can be seen that, as expected, the active/active configuration exhibits both less displacement along both the $X_{E}$ and $Y_{E}$ axes than any of the other configurations. Conversely, the open/open configuration, with the less combined grip than the other configurations (see Figure 3), 
takes the outermost path. It is also interesting to compare the speed histories in Figure 7. a to the utilization of the combined grip in Figure 7. b. There it can be seen that in particular between the active/active and the open/open configuration, the difference is notable in the combined grip area. The active/active configuration has the steepest changes in longitudinal speed and does also reach the lowest speed of all configurations. These rapid changes in speed explain why the trajectory has the highest average curvature.

Note that these results intend to show the maximum performance of each configuration, not necessarily the desired trajectory. If all vehicles are, for example, to have the same (feasible) speed profile, the difference in the configurations would instead be a difference in friction utilization and thereby a larger safety margin for the more capable configuration.

Although not predicted by the QSS approximation, it can be seen that the highest lateral acceleration is achieved for the open/open configuration. This is likely due to that the time at which the speed is constant (which is when the maximum lateral acceleration is achieved) is much shorter for the active/active configuration than for the open/open configuration. It is suggested that for the active/active configuration, the vehicle never reaches steady-state. This would indicate that, in this example, the QSS assumption is likely not entirely valid during the entire range of combined grip operated in this example. Nevertheless, given the simplicity with which the drive force distribution can be optimized, and that the qualitative outcome of the vehicle simulation is well predicted by the QSS method, it is still considered useful for these type of situations.

\section{Summary}

The purpose of this study was to study the optimal force distribution for combined traction/braking and cornering with the objective to maximize the combined lateral and longitudinal grip in a particular direction. The aim was to find a simple and flexible problem formulation for the present problem.

These objectives were achieved by formulating the problem as a quadratically constrained linear programming (QCLP) problem based on the quasi steady state (QSS) assumption. The QCLP problem was solved for four different driveline configurations with different constraints on the left/right drive force distribution. The QCLP formulation allowed for these configurations to be easily implemented without reformulation of the optimization problem for each case. Further, some examples were given how the front or rear wheel drive configurations can be implemented as well, further demon- 
(a)
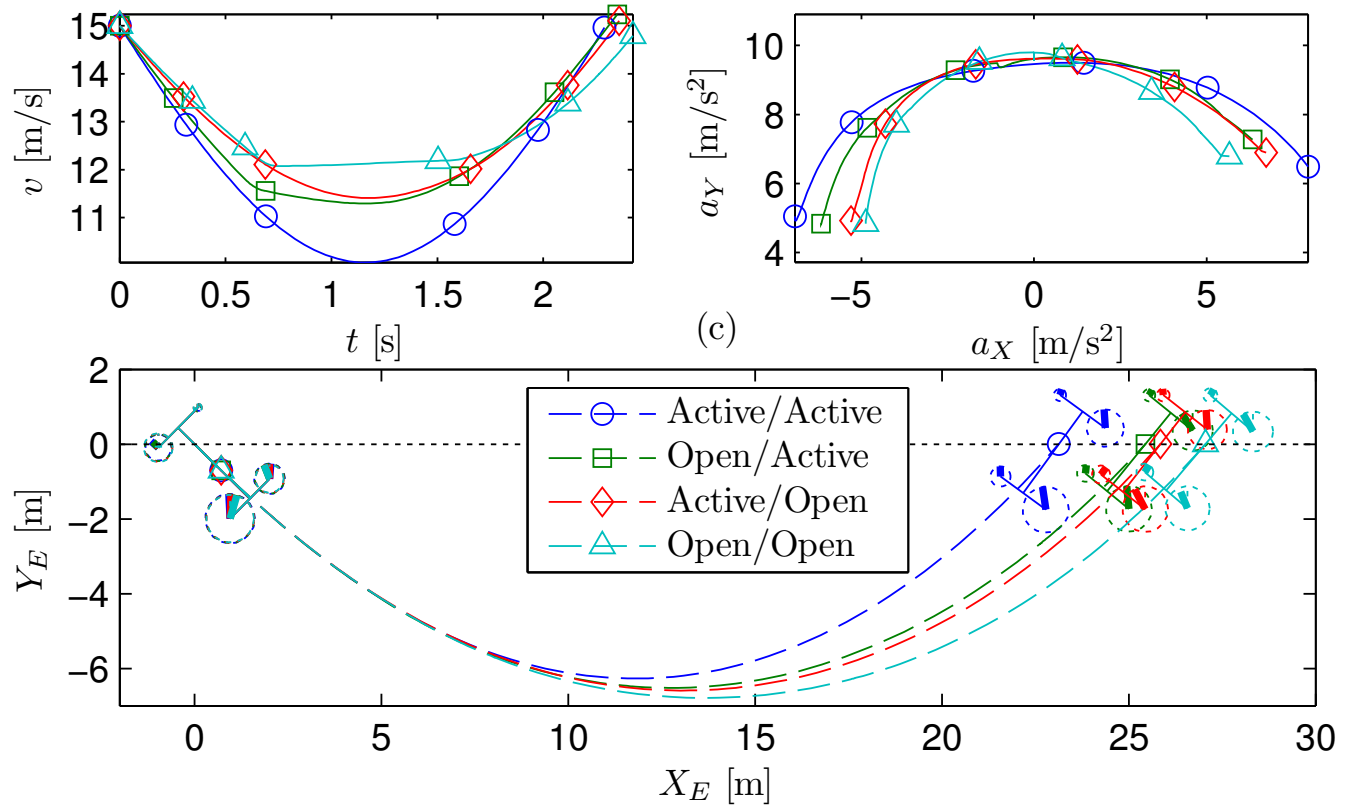

(b)

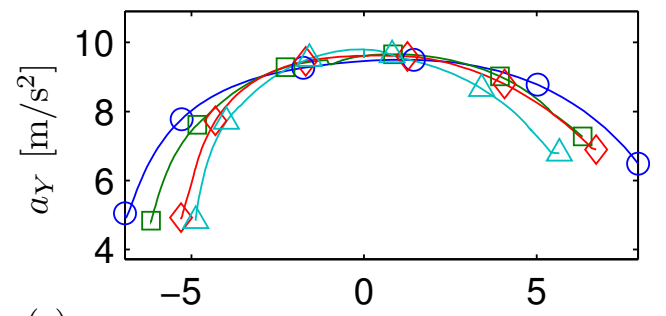

(c)

Figure 7: Results from vehicle implementation of the longitudinal force distribution obtained from the QCLP optimization. The target global force direction is fixed (upward) in the inertial reference frame. 
strating the flexibility of the chosen approach.

Additionally, a closed-form solution to the general optimization problem was given for the case where the force distribution can be individually controlled. These derived expressions are for this special case, equivalent to the constrained optimization problem, but being in a much simpler form.

Also a new linear approximation of the quadratic constraints was presented, which reduces the nonlinear problem to a standard linear programming problem. This formulation may have applications in on-line optimization or linear model predictive control (MPC).

Finally an example implementation of the off-line computed optimization results in the presented vehicle model are shown to yield results closely following the predictions from the QSS optimization.

In conclusion, this study has demonstrated the application of the QCLP formulation for the study of driveline configurations for the QSS approximation of cornering problems involving combined longitudinal and lateral acceleration near the vehicle limit. It is straightforward to extend the proposed method to braking, and it is not unlikely that also other applications, such as active steering, are possible.

The optimization of the distribution of the individual wheel forces using the QSS assumption provides a tool to understand the implications of particular driveline configurations on the combined lateral and longitudinal grip envelope [16, 17]. The addition of the QCLP problem formulation by this study, makes another powerful tool available to the vehicle dynamics analyst to perform such studies.

\section{Acknowledgments}

The author wishes to thank the Swedish national research program Intelligent Vehicle Safety Systems (IVSS) for financial support. Additionally to Anders Boström, Bo Egardt and Mathias Lidberg from Chalmers University of Technology and Gunnar Olsson from Saab Automobile for supervision of this work. Also Tim Gordon from the University of Michigan Transportation Research Institute provided very valuable feedback on this work. All figures are exported from Matlab ${ }^{\mathrm{TM}}$ using print_pdf by Oliver Woodford.

\section{References}

[1] M. Abe. A theoretical analysis on vehicle cornering behaviors in acceleration and in braking. In 9th IAVSD Symposium, Dynamics of Vehicles 
on Roads and on Tracks, 1986.

[2] M. Abe. Effects of traction force distribution control with additional rear wheel steer on turning behavior of $4 \mathrm{wd}$ vehicle. Vehicle System Dynamics, 17(6 supp 1):1-12, 1988.

[3] M. Abe. Vehicle Handling Dynamics: Theory and Application. Butterworth Heinemann, Oxford, 2009.

[4] J. Andreasson. On Generic Road Vehicle Motion Modelling and Control. PhD thesis, Royal Institute of Technology - Department of Aeronautical and Vehicle Engineering, 2006.

[5] J. Backmark, E. Karlsson, J. Fredriksson, and M. Jonasson. Using future path information for improving the stability of an over-actuated vehicle. International Journal of Vehicle Systems Modelling and Testing, 4:3, s. 218-231, 2009.

[6] C. Borroni-Bird and M. Shabana. Chevrolet sequel: Reinventing the automobile. In SAE Technical Paper 2008-01-0421, 2008.

[7] S. Boyd and L. Vandenberghe. Convex Optimization. Cambridge University Press, Cambridge, 2004.

[8] D. Brayshaw and M. Harrison. A quasi steady state approach to race car lap simulation in order to understand the effects of racing line and centre of gravity location. Proceedings of the Institution of Mechanical Engineers, Part D: Journal of Automobile Engineering, 219(6):725-739, Jan. 2005.

[9] P. Falcone, H. Eric Tseng, F. Borrelli, J. Asgari, and D. Hrovat. Mpcbased yaw and lateral stabilisation via active front steering and braking. Vehicle System Dynamics, 46(1 supp 1):611-628, 2008.

[10] M. Jonasson. Exploiting individual wheel actuators to enhance vehicle dynamics and safety in electric vehicles. PhD thesis, KTH, Aeronautics and Vehicle Engineering, 2009.

[11] M. Jonasson and J. Andreasson. Exploiting autonomous corner modules to resolve force constraints in the tyre contact patch. Vehicle System Dynamics, 46(7):553-573, 2008.

[12] M. Klomp. On Drive Force Distribution and Road Vehicle Handling A Study of Understeer and Lateral Grip. Licentiate Thesis, Chalmers University of Technology, 2007. 
[13] L. Laine. Reconfigurable Motion Control Systems for Over-Actuated Road Vehicles. PhD thesis, Chalmers University of Technology, Department of Applied Mechanics, 2007.

[14] E. Ono, Y. Hattori, Y. Muragishi, and K. Koibuchi. Vehicle dynamics integrated control for four-wheel-distributed steering and fourwheel-distributed traction/braking systems. Vehicle System Dynamics, 44(2):139-151, February 2006.

[15] H. B. Pacejka. Tyre and Vehicle Dynamics. Butterworth-Heinemann, Oxford, UK, 2 edition, 2006.

[16] H. Peng and J.-S. Hu. Traction/braking force distribution for optimal longitudinal motion during curve following. Vehicle System Dynamics, 26(4):301-320, 1996.

[17] K. Sawase and Y. Ushiroda. Improvement of vehicle dynamics by rightand-left torque vectoring system in various drivetrains. Mitsubishi Technical Review, 20:14-20, 2008.

[18] F. Uffelmann. Automotive stability and handling dynamics in cornering and braking maneuvers. Vehicle System Dynamics, 12(4\&5):203-223, 1983.

[19] H.-P. Willumeit, M. Neculau, A. Vikas, and A. Whler. Mathematical models for the computation of vehicle dynamic behaviour during development. In Proceedings of the 24th FISITA Congress, 1992.

[20] H. Yoshida, S. Shinohara, and M. Nagai. Lane change steering manoeuvre using model predictive control theory. Vehicle System Dynamics, 46(1 supp 1):669-681, 2008.

\section{A Vehicle and Tire Data}

The vehicle data in Table 1 is used in the conducted simulations are taken from [17] and represent a medium-sized passenger vehicle. The yaw inertia was not available in [17] but is taken from another vehicle with similar size. 


\begin{tabular}{|l|c|c|c|}
\hline Description & Variable(s) & Unit & Value(s) \\
\hline \hline Vehicle mass (front/rear) & $m_{1} / m_{2}$ & {$[\mathrm{~kg}]$} & $900 / 600$ \\
\hline Yaw radius of gyration & $k$ & {$[\mathrm{~m}]$} & $\sqrt{15 / 26}$ \\
\hline Wheel base & $l$ & {$[\mathrm{~m}]$} & 2.7 \\
\hline Height of mass center & $h$ & {$[\mathrm{~m}]$} & 0.5 \\
\hline Track width (f/r) & $w$ & {$[\mathrm{~m}]$} & 1.5 \\
\hline Lateral load transfer coefficient (f/r) & $\zeta_{Y 1} / \zeta_{Y 2}$ & {$[-]$} & $0.17 / 0.16$ \\
\hline \hline Axle friction (f/r) & $\mu_{1} / \mu_{2}$ & {$[-]$} & $1.0 / 1.1$ \\
\hline Tire shape factor & {$[-]$} & $C$ & $3 / 2$ \\
\hline Tire stiffness factor & {$[-]$} & $B$ & 10 \\
\hline
\end{tabular}

Table 1: Vehicle and tire data 